\title{
Aspectos ambientais relacionados à síntese, utilização e descarte do grafeno
}

\section{Paulo Gabriel Ferreira de Azevedo, Caio de Oliveira Pinto $^{1}$, Luana Kelly de Jesus Santos ${ }^{1}$, Ivan Costa da Silva ${ }^{1}$, Cláudia Teresa Teles Farias ${ }^{1}$ e Luiz Antônio Pimentel Cavalcanti $^{2}$}

\author{
${ }^{1}$ Instituto Federal de Educação, Ciência e Tecnologia da Bahia. Curso de Engenharia \\ Química. Campus Salvador. Rua Emídio dos Santos, S/№. Bairro do Barbalho. \\ Salvador-BA, Brasil (CEP 40301-015). \\ ${ }^{2}$ Instituto Federal de Educação, Ciência e Tecnologia da Bahia. Campus Salvador. \\ Rua Emídio dos Santos S/№. Bairro de Barbalho. Salvador-BA, Brasil (CEP 40301- \\ 015). E-mail: luiz.cavalcanti@ifba.edu.br.
}

\begin{abstract}
Resumo. O grafeno vem ganhando notoriedade e sendo amplamente estudado e aplicado devido às suas excelentes propriedades físicas e químicas; com isso, à medida que se aumenta o interesse na sua utilização, cresce também a necessidade de compreender como este nanomaterial relaciona-se com o meio ambiente, posto à importância do desenvolvimento sustentável. Para tal, este artigo apresenta uma revisão integrativa da literatura sobre o grafeno e seus derivados, objetivando abordar as seguintes frentes: (i) resíduos gerados na produção do grafeno e como estes rejeitos podem impactar o meio ambiente, bem como sugestões de gerenciamento destes visando à redução dos impactos, (ii) após sua produção, a forma como o grafeno pode ser aplicado, de modo a tornar os processos ambientalmente mais amigáveis, e, por fim, (iii) como o descarte irregular desse produto, após o seu uso, pode impactar o meio ambiente. A revisão foi realizada selecionando trabalhos que abordassem o grafeno e seus derivados nas instâncias supracitadas. Desses trabalhos, pôde-se observar que (i) a síntese do grafeno gera resíduos que podem ser devidamente tratados, com processos de purificação, extração, entre outros; (ii) há uma ampla aplicação do grafeno no setor energético, sobretudo, o solar, além do seu uso como adsorventes em tratamentos de efluentes; e, por fim (iii) notou-se que os resíduos de grafeno (e de outros nanomateriais de carbono) podem impactar negativamente o meio ambiente (seja aquático, no solo ou no ar), tornando necessárias políticas de controle.
\end{abstract}

Palavras-chave: Grafeno; Resíduos; Aplicações ambientais; Impactos ambientais.
Recebido

$07 / 06 / 2021$

Aceito

$26 / 07 / 2021$

Disponível on line

$31 / 07 / 2021$

Publicado

$31 / 08 / 2021$

Acesso aberto

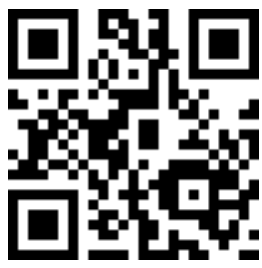

() 0000-0003-0685-2928 Paulo Gabriel Ferreira de Azevedo

(ㄷ) 0000-0001-8021-2430 Caio de Oliveira Pinto 
Abstract. Environmental aspects related to the graphene synthesis, utilization and disposal. Graphene has been gaining notoriety and has been extensively studied and applied due to its excellent physical and chemical properties; so, as interest in the use of graphene increases, also increases the necessity to understand how this carbon nanomaterial relates to the environment, seeing the importance of sustainable development. To this end, this article presents an integrative literature review about graphene and derivatives carried out aiming to address the following fronts: (i) residues generated in the production of graphene and how these residues can impact the environment, as well as suggestions for their management aiming at reducing impacts, (ii) after its production, the way in which graphene can be applied, in order to make the processes more environmentally friendly, and, finally, (iii) how the irregular disposal of this technology, after its use, can impact the environment. This review was done selecting works that approached graphene and derivatives in the aforementioned instances. From these works, it can be observed: (i) graphene synthesis generates residues that can be properly treated, with processes of purification, extraction, among others; (ii) there is an ample application of graphene in the energy sector, especially in the solar sector, beyond its use as adsorbents in effluent treatments; and finally (iii) it was observed that graphene residues (and other carbon nanomaterials) can negatively impact the environment (whether aquatic, on the ground or in the air), making control policies necessary.

Keywords: Graphene; Residues; Environmental applications; Environmental impacts.
(ㄱ) $0000-0002-7092-647 \mathrm{X}$

Luana Kelly de Jesus

Santos

(1) 0000-0001-5990-5179

Ivan Costa da Silva

(1) 0000-0002-3976-613X

Cláudia Teresa Teles

Farias

(D) 0000-0003-4932-9387

Luiz Antônio Pimentel

Cavalcanti

\section{Introdução}

Considerado como o material do futuro, o grafeno tem sido amplamente estudado em distintas frentes de estudo, tais como a química, biologia, ciências dos materiais, nanociência entre outras áreas de conhecimento (Marion e Hasan, 2016). De acordo com Silva (2016), inicialmente o grafeno foi descoberto em 1962, mas a descoberta não foi levada adiante; somente em 2004 os físicos Kostya Novoselov e Andre Gein lideraram uma equipe que trouxeram esse material para o cenário científico mundial.

O grafeno é uma camada (2D) de átomos de carbono ligados entre si com hibridização $\mathrm{sp}^{2}$ (Oliveira, 2014). De acordo com a Teoria do Orbital Molecular, a hibridização $\mathrm{sp}^{2}$ é formada pela combinação linear entre os orbitais $s, \mathrm{p}_{\mathrm{x}}$ e $\mathrm{p}_{\mathrm{y}}$, sendo os dois últimos pertencentes aos orbitais do tipo p. Assim, a hibridização $\mathrm{sp}^{2}$ contribui para que os carbonos, no grafeno, formem uma estrutura trigonal plana, com ligações do tipo sigma $(\sigma)$; dessa geometria, tem-se que o orbital $p_{z}$, orbital do tipo p que não se combinou com o $\mathrm{s}$ para formar $\mathrm{o} \mathrm{sp}^{2}$ e que é perpendicular a estrutura trigonal, tenha um elétron livre, de simetria pi $(\pi)$ e fora do plano trigonal, sendo que esse elétron liga-se mais fracamente do que os elétrons de simetria $\sigma$. Assim, esses elétrons livres de simetria $\pi$ conferem ao grafeno propriedades elétricas e térmicas diferenciadas, quando comparadas com outros materiais, como o silício (Oliveira, 2014; Vieira Segundo e Vilar, 2016). 
Devido às suas configurações químicas, o grafeno tem sido cada vez mais explorado, tanto nas instâncias experimentais, como de aplicações tecnológicas. Sua superfície é próxima do inerte, sendo que para que o grafeno tenha interação química, introduz-se defeitos ou espécies reativas (Vieira Segundo e Vilar, 2016). É considerado fino, leve, de alta flexibilidade e de alta barreira para gases, dificultando a difusão desses (Costa, 2017). Altas condutividades térmica (grafeno $\approx 5000 \mathrm{Wm}^{-1} \cdot \mathrm{K}^{-1}$, cobre $\approx 390 \mathrm{Wm}^{-1} \cdot \mathrm{K}^{-1}$ ) e elétrica (mobilidade elétrica $\approx 200.000 \mathrm{~cm}^{2} \cdot \mathrm{V}^{-1} \cdot \mathrm{s}^{-1}$ ), sendo a última cerca de 100 vezes maior que a do silício, e sua alta área superficial $\left(2630 \mathrm{~m}^{2} \cdot \mathrm{g}^{-1}\right)$ aliada aos elétrons livres de simetria $\pi$ conferem ao grafeno interessantes características adsortivas (Silva, 2016). Além disso, possui módulo de Young (grafeno $\approx 1 \mathrm{TPa}$, aço $\approx 0,2 \mathrm{TPa}$ ) $\mathrm{e}$ resistência a tração elevados (grafeno $\approx 125 \mathrm{GPa}$, aço $\approx 1,2 \mathrm{GPa}$ ) (Vieira Segundo e Vilar, 2016; Costa, 2017).

Apesar do notório avanço tecnológico que o grafeno proporciona, pesquisadores preocupam-se em como esse material irá se comportar frente às necessidades ambientais, posto que a sustentabilidade tem sido fortemente discutida ao redor do mundo. Assim, visando a diminuir, ou a sanar, os impactos ambientais causados pelas atividades antrópicas, torna-se interessante o estudo de como o grafeno aborda essa temática. Um dos chamados Objetivos de Desenvolvimento do Milênio, acordados pelos países-membros da Organização das Nações Unidas em 2000, é o comprometimento em garantir o desenvolvimento sustentável, isto é, que os avanços científicos e tecnológicos seriam ambientalmente amistosos (Roma, 2019). Portanto, o presente trabalho visa a levantar aspectos que relacionam a pesquisa/aplicação do grafeno no cenário do meio ambiente, pontuando facetas nas quais o grafeno se relaciona com temas ambientais.

\section{Metodologia}

No presente trabalho, propõe-se a ser uma revisão integrativa cruzando trabalhos já desenvolvidos sobre grafeno e meio ambiente. Assim, foram realizadas buscas nas plataformas "Google Scholar", "Scielo" e "Science Direct", utilizando os descritores "grafeno", "síntese de grafeno", "aplicações do grafeno" e "desenvolvimento sustentável grafeno", nos idiomas português e inglês ("graphene", "graphene sinthesis" e "graphene applications"), com recorte temporal entre 2010 a 2021. Como critério de inclusão, foram selecionados artigos que possuem resultados sobre o grafeno correlacionando com o meio ambiente, sob os aspectos mencionados anteriormente. Foram excluídos artigos que somente abordavam o grafeno sem o aspecto ambiental ou que divergissem do tema. Assim, foram selecionados 10 artigos que abordaram os resíduos gerados pela síntese do grafeno (etapa i), 8 artigos sobre aplicação do grafeno nas vertentes ambientais (etapa ii) e 13 artigos no tocante ao descarte do grafeno após seu uso (etapa iii), totalizando 31 trabalhos, sendo as suas respectivas referências descritas na Tabela 1. No total, foram investigados 54 trabalhos nos quais o tema principal é o grafeno, portanto 23 foram excluídos por não contemplarem os requisitos. 
Tabela 1. Referências dos trabalhos incluídos neste trabalho.

\begin{tabular}{|c|c|c|}
\hline Etapa & Referência & Objetivo \\
\hline \multirow{10}{*}{ (i) } & $\begin{array}{l}\text { Camargos et al. } \\
\text { (2017) }\end{array}$ & $\begin{array}{l}\text { Realizar revisão sobre formas de sintetizar o grafeno, bem como } \\
\text { os principais métodos para sua caracterização, e aplicações. }\end{array}$ \\
\hline & Castro (2011) & $\begin{array}{l}\text { Sintetizar o grafeno pelo método CVD visando a verificar e a } \\
\text { aperfeiçoar os resultados existentes na literatura. }\end{array}$ \\
\hline & $\begin{array}{l}\text { Coletti et al. } \\
\text { (2019) }\end{array}$ & $\begin{array}{l}\text { Analisar as vantagens da recuperação da acetona em comparação } \\
\text { com sua incineração. }\end{array}$ \\
\hline & $\begin{array}{l}\text { Cordeiro et al. } \\
\quad(2015)\end{array}$ & Realizar síntese química e caracterização do grafeno. \\
\hline & Leal (2016) & $\begin{array}{l}\text { Analisar a condutividade elétrica de camadas de grafeno obtidas } \\
\text { por esfoliação mecânica. }\end{array}$ \\
\hline & Martinez (2018) & $\begin{array}{l}\text { Estudar a fixação de gases de combustão do sistema motogerador } \\
\text { por meio de cultivo de microalgas. }\end{array}$ \\
\hline & Nunes (2019) & $\begin{array}{l}\text { Estudar parâmetros reacionais visando à obtenção de grafeno } \\
\text { com maior estabilidade térmica e estrutural. }\end{array}$ \\
\hline & $\begin{array}{l}\text { Rodrigues et al. } \\
\text { (2010) }\end{array}$ & $\begin{array}{l}\text { Sugerir o gerenciamento, e possíveis tratamentos de resíduos } \\
\text { químicos. }\end{array}$ \\
\hline & Sá (2011) & $\begin{array}{l}\text { Investigar o processo de crescimento epitaxial de grafeno, com } \\
\text { posterior caracterização do mesmo. }\end{array}$ \\
\hline & $\begin{array}{l}\text { Vidales et al. } \\
\text { (2013) }\end{array}$ & $\begin{array}{l}\text { Estudar a viabilidade de reciclagem de resíduos poliméricos para } \\
\text { a produção de blendas. }\end{array}$ \\
\hline \multirow{9}{*}{ (ii) } & Araújo (2018) & $\begin{array}{l}\text { Analisar a produção de aplicação do grafeno para a remoção de } \\
\text { contaminantes em meio aquoso. }\end{array}$ \\
\hline & Bao et al. (2016) & $\begin{array}{l}\text { Investigar a capacidade adsortiva de miçangas de grafeno na } \\
\text { limpeza de produtos químicos envolvidos em vazamento. }\end{array}$ \\
\hline & Chen et al. (2013) & $\begin{array}{l}\text { Estudar sobre o grafeno e seus derivados para o desenvolvimento } \\
\text { de painéis solares e aplicações fotoeletroquímicas e } \\
\text { fotocatalíticas. }\end{array}$ \\
\hline & Ferreira (2015) & $\begin{array}{l}\text { Estudar o desenvolvimento de materiais lignocelulósicos e } \\
\text { quitinosos para aplicação em química ambiental. }\end{array}$ \\
\hline & $\begin{array}{l}\text { Huang et al. } \\
\text { (2017) }\end{array}$ & $\begin{array}{l}\text { Sugerir a substituição do eletrodo auxiliar das células solares } \\
\text { sensibilizadas por corante por um eletrodo de } \mathrm{MoS}_{2} / \text { papel grafite. }\end{array}$ \\
\hline & Lai et al. (2019) & $\begin{array}{l}\text { Estudar a aplicação do grafeno em materiais tridimensionais } \\
\text { objetivando a adsorção de tintas e metais pesados. }\end{array}$ \\
\hline & Lima et al. (2020) & $\begin{array}{l}\text { Analisar a adsorção de azul de metileno frente à hidrocarvões de } \\
\text { resíduos têxteis. }\end{array}$ \\
\hline & $\begin{array}{l}\text { Martins et al. } \\
\text { (2018) }\end{array}$ & $\begin{array}{l}\text { Elaborar modelos computacionais para estudar a aplicação de } \\
\text { nanocompósitos de } \mathrm{TiO}_{2} / \text { Grafeno em aplicações fotocatalíticas. }\end{array}$ \\
\hline & Rocha (2015) & $\begin{array}{l}\text { Estudar sobre a utilização em longo prazo do grafeno para } \\
\text { produção de sistemas de energia limpa, alta eficiência e baixo } \\
\text { custo. }\end{array}$ \\
\hline \multirow{6}{*}{ (iii) } & $\begin{array}{l}\text { Ferreira et al. } \\
\quad(2019)\end{array}$ & $\begin{array}{l}\text { Estudar a toxicidade do OG com e sem ativação por } \mathrm{NH}_{2} / \mathrm{Fe} \text { frente } \\
\text { a sementes de Lactuca sativa L. }\end{array}$ \\
\hline & $\begin{array}{l}\text { Ferreira et al. } \\
\qquad(2015)\end{array}$ & $\begin{array}{l}\text { Comparar os efeitos do resíduo do bagaço de cana queimado, } \\
\text { nanoestrutura de carvão ativado e grafeno comercial frente ao } \\
\text { bioindicador Hydra attenuata. }\end{array}$ \\
\hline & Hu e Zhou (2013) & $\begin{array}{l}\text { Estudar a relação entre o grafeno, a saúde humana e o meio } \\
\text { ambiente. }\end{array}$ \\
\hline & Hu et al. (2014) & Analisar os impactos da combinação entre As e grafeno no trigo. \\
\hline & $\begin{array}{l}\text { Katwa et al. } \\
\text { (2012) }\end{array}$ & $\begin{array}{l}\text { Estudar como mastócitos pulmonares e cardiovasculares reagem } \\
\text { à exposição de nanotubos de carbono. }\end{array}$ \\
\hline & $\begin{array}{l}\text { Rhiem et al. } \\
\text { (2016) }\end{array}$ & $\begin{array}{l}\text { Quantificar a liberação de resíduos de nanotubos de carbono pelo } \\
{ }^{14} \mathrm{C} .\end{array}$ \\
\hline
\end{tabular}


Tabela 1. Continuação.

\begin{tabular}{|c|c|l|}
\hline Etapa & Referência & Objetivo \\
\hline \multirow{5}{*}{ (iii) } & $\begin{array}{c}\text { Schlagenhauf et al. } \\
(2014)\end{array}$ & $\begin{array}{l}\text { Realizar uma revisão sobre deposição de nanotubos de carbono } \\
\text { por diversas ações naturais simuladas. }\end{array}$ \\
\cline { 2 - 4 } & $\begin{array}{c}\text { Seabra et al. } \\
(2013)\end{array}$ & $\begin{array}{l}\text { Investigar o uso do ácido sulfúrico e o peróxido de hidrogênio na } \\
\text { degradação de catalisadores residuais impregnados em } \\
\text { nanotubos de carbono. }\end{array}$ \\
\cline { 2 - 4 } & Song et al. (2021) & $\begin{array}{l}\text { Avaliar como a concentração de OG no solo impacta no } \\
\text { crescimento da Larix olgensis. }\end{array}$ \\
\cline { 2 - 4 } & Zhang et al. (2017) & $\begin{array}{l}\text { Analisar as consequências da exposição do OG aos embriões do } \\
\text { peixe-zebra Danio rerio. }\end{array}$ \\
\cline { 2 - 4 } & Zhao et al (2017) & $\begin{array}{l}\text { Investigar a toxicidade de três tipos de grafeno frente à alga } \\
\text { Chlorella pyrenoidosa. }\end{array}$ \\
\cline { 2 - 4 } & $\begin{array}{c}\text { Zarbin e Oliveira } \\
\text { (2013) }\end{array}$ & $\begin{array}{l}\text { Analisar o mercado de nanotubos de carbono, novas descobertas } \\
\text { científicas e desafios, ambientais e econômicos, conferidos por } \\
\text { essa tecnologia. }\end{array}$ \\
\hline
\end{tabular}

\section{Resultados e discussão}

Nos últimos anos, o número de pesquisas sobre o grafeno cresceu consideravelmente. Nesse estudo, foram investigados e incluídos 31 trabalhos, com três perspectivas distintas acerca deste nanomaterial. Inicialmente, aborda-se sua síntese e seus principais resíduos.

\section{Resíduos gerados na síntese do grafeno}

0 grafeno pode ser obtido por diversas rotas; por conta disso, para a realização de sua síntese, deve-se considerar o nível de qualidade e quantidade requeridas para dada aplicação, e a viabilidade econômica do processo. A obtenção do grafeno pode se dar pelos processos de bottom-up e top-down. 0 processo bottom-up consiste na obtenção do grafeno a partir de moléculas menores, sendo os principais métodos o de Crescimento Epitaxial e o de Deposição Química de Vapor (CVD). Já o processo top-down consiste na obtenção do grafeno por meio da fragmentação do grafite, sendo os principais métodos o de Separação Mecânica e o de Separação Química (Camargos et al., 2017)

Utilizando o método de crescimento epitaxial, Sá (2011) sintetizou grafeno com o objetivo de analisar como se daria o crescimento das multicamadas de grafeno sob um substrato à pressões atmosféricas. Por existirem poucos trabalhos a respeito da obtenção de grafeno nestas condições, e sabendo que o grafeno obtido a pressão atmosférica apresenta melhorias em sua morfologia em comparação ao obtido em ultra alto vácuo, o autor submeteu o cristal de carbeto de silício $(\mathrm{SiC})$ à altas temperaturas em presença de Argônio, promovendo a liberação de $\mathrm{Si}$ da superfície do cristal, e reorganização dos átomos de carbono, levando à formação de camadas de grafeno que se sobrepõem à medida que os átomos de silício vão sendo sublimados. Após a síntese, o produto obtido foi analisado através de Espectroscopia Raman.

Com base nos resultados obtidos, onde poucos defeitos foram encontrados no material sintetizado, o autor concluiu que, por suas características condutoras, a utilização do nanomaterial é vantajosa em determinadas aplicações na área da eletrônica, bem como não há a necessidade de transferência de substrato, pois como o SiC é um isolante, este pode utilizado juntamente com o grafeno. Ao final do processo, foram gerados resíduos de acetona e álcool isopropílico, solventes utilizados na limpeza inicial do substrato por ultrassom. 
Os resíduos gerados no método de crescimento epitaxial, de acetona e álcool isopropílico, são materiais inflamáveis cuja disposição final geralmente é a incineração, levando à geração de resíduos de queima e emissão de gases de efeito estufa (GEE), e impactando o meio ambiente. Visando à minimização dos impactos gerados por estes resíduos, Coletti (2019) sugere a destilação de ambos, pois dessa forma haveria a recuperação dos solventes, possibilitando sua reutilização, e minimização dos resíduos gerados e gases emitidos, sendo assim um tratamento mais sustentável.

Já no método de deposição química de vapor (CVD), Castro (2011) visou a sintetizar o grafeno com o objetivo de analisar os dados pré-existentes na literatura, bem como relacionar as variáveis de análise com as propriedades do grafeno produzido. Para isso, foi realizada a decomposição de um fluxo gasoso de $\mathrm{CH}_{4} \mathrm{em}$ altas temperaturas, na presença do substrato $\mathrm{Si} / \mathrm{SiO}_{2}$ revestido do catalisador $\mathrm{Ni}$, levando a formação de lâminas de grafeno sobre o metal. Este procedimento foi realizado em diferentes condições reacionais, e os resultados obtidos revelaram que o grafeno foi sintetizado com qualidade em ambas as condições, mas apresentava defeitos em diferentes proporções.

Por ser um processo de decomposição, os resíduos significativos gerados ao final da síntese são decorrentes da transferência do filme de grafeno para outro substrato, como o resíduo da solução aquosa de ácido clorídrico $(\mathrm{HCl})$ utilizada para atacar o $\mathrm{Ni}$, a acetona e o polimetilmetacrilato (PMMA), que não podem ser dispensados diretamente no meio ambiente, já que a solução ácida de cloreto de níquel é nociva aos organismos aquáticos e seres humanos, pois o Níquel é um metal tóxico e acumulativo (RODRIGUES et al., 2010), a acetona é inflamável e geralmente destinada à incineração, levando a emissão de GEE e geração de resíduos provenientes da queima (Coletti, 2019) e o PMMA é um resíduo polimérico sólido e não biodegradável (Vidales et al., 2013).

Com base nestas informações, são propostas formas de reduzir os impactos causados pela geração destes resíduos químicos através de um tratamento adequado dos mesmos. No caso da solução ácida de cloreto de níquel, faz-se necessária a precipitação do sal em forma de hidróxido de níquel, que por conta de suas propriedades toxicológicas não deve ser descartado como efluente, mas deve ser eliminado na forma sólida (Rodrigues et al., 2010); sugere-se a destilação da acetona, possibilitando assim que a mesma seja reutilizada, e os resíduos finais gerados sejam reduzidos (Coletti, 2019); e o polímero PMMA pode ser encaminhado para reciclagem, onde pode ser reutilizado, a exemplo de sua aplicação na produção de blendas (Vidales et al., 2013).

0 grafite é constituído de diversas camadas de grafeno, que podem ser separadas por meio de métodos de separação. Através do método de separação mecânica, Leal (2016) promoveu a esfoliação destas camadas por meio de uma fita adesiva e ação mecânica, por meio de repetições sucessivas do procedimento de "cola e descola", e as transferiu para o substrato $\mathrm{Si} / \mathrm{SiO}_{2}$ mecanicamente, com o objetivo de avaliar a condutividade elétrica apresentada por poucas camadas de grafeno. 0 autor concluiu, por meio dos resultados obtidos, que a separação mecânica, bem como as propriedades apresentadas pelo grafeno, foram satisfatórias. Este é um método simples de obtenção do grafeno, e por consistir em sua obtenção de forma mecânica utilizando somente fita adesiva, não há geração de resíduos ao final do processo, apresentando menor impacto ao meio ambiente. Em contrapartida, as quantidades de grafeno obtidas são pequenas, não sendo viável para a produção em larga escala.

Já Nunes (2019) estudou a obtenção do grafeno mediante a separação química, pretendendo definir as melhores condições para a obtenção do grafeno com maior estabilidade estrutural e térmica. 0 autor, mediante a promoção da oxidação do grafite, com ácido sulfúrico concentrado, permanganato de potássio $\left(\mathrm{KMnO}_{4}\right)$ e nitrato de sódio, obteve óxido de grafeno (OG), como exibido na Equação 1. 


$$
\left.n(\text { grafite })+n \mathrm{H}_{2} \mathrm{SO}_{4}+n / 2 \text { [oxidante }\right] \rightarrow n\left[\text { grafite }-\mathrm{HSO}_{4}\right]+n / 2 \mathrm{H}_{2} \mathrm{O} \text { (Equação } 1 \text { ) }
$$

Posteriormente, foram utilizados peróxido de hidrogênio, ácido clorídrico e água destilada, para promover a purificação do OG sintetizado. Em seguida, foi realizada uma etapa de redução térmica sob diferentes temperaturas, para eliminação das interferências causadas pelos radicais presentes. Ao fim o autor concluiu que, mediante os métodos de síntese e redução adotados, foi possível obter um material com alta estabilidade.

Utilizando o método de separação química, além dos resíduos gerados pelo excesso de reagentes utilizado, e dos aplicados na etapa de lavagem, ocorre a liberação dos gases tóxicos dióxido de nitrogênio $\left(\mathrm{NO}_{2}\right)$ e tetróxido de nitrogênio $\left(\mathrm{N}_{2} \mathrm{O}_{4}\right)$ na redução do grafeno, gerando impactos ao meio ambiente (Cordeiro et al., 2015). Objetivando dar uma destinação a estes resíduos de forma a minimizar os danos ao meio ambiente, Rodrigues et al. (2010) sugere que a solução ácida residual, ao depender do pH apresentado, deve ser diluída ou neutralizada, e encaminhada para efluente; e os gases tóxicos liberados na redução do grafeno podem ser capturados por meio da utilização de microalgas, onde são transformados em nutrientes, reduzindo assim sua emissão e poluição do ar (Martinez, 2018).

\section{Aplicações do grafeno para o meio ambiente}

Vê-se então os resíduos gerados pelos processos de síntese do grafeno, e as formas de lidar com o descarte, mas ao mesmo tempo há benefícios na utilização do grafeno quando trata-se do campo ambiental, podendo ser utilizado em âmbito energético, tratamento de efluentes, melhoria na eficiência de células solares, fotocatálise, entre outras aplicações.

No que se refere à energia, a utilização do OG pode ser destinada à construção de supercapacitores, substituindo os metais pesados que são utilizados atualmente, além de ser aplicável a sistemas de energia renovável e na melhoria da eficiência de outros, sendo então uma tentativa de suprir a alta demanda energética ocasionada pelo crescimento populacional e avanço tecnológico (Rocha, 2015).

De acordo com Chen et al. (2013), a sua aplicação em células fotovoltaicas orgânicas atuando como eletrodos transparentes, aceptor de elétrons e "hole transporters" pode acarretar em aumento da performance e maior eficiência na conversão de energia, um fator que supera a maior dificuldade na utilização desse tipo de célula. A possibilidade de uso do grafeno como eletrodos transparentes supera a problemática de escassez do atual material utilizado, o índio, presente no óxido de índio-estanho (ITO) que a cada vez fica mais caro, a substituição do ITO pelo grafeno torna a transmissão física e a condutividade mais eficazes.

Prova-se ainda sua utilização em células solares sensibilizadas por corante (CSSCs), que são alternativas promissoras para fontes alternativas, mas que sua utilização ainda depende da síntese de dióxido de titânio $\left(\mathrm{TiO}_{2}\right)$ e o custo alto do eletrodo auxiliar de platina; nesse âmbito, o grafeno tornou-se promissor para a substituição de diversos componentes presentes nessas células, mostrando-se eficiente em performance e possibilitando um bom custo benefício durante a fabricação das mesmas (Chen et al, 2013). De acordo com o artigo de Huang et al. (2017), CSSCs de baixo custo e alta eficiência podem ser produzidas substituindo o eletrodo auxiliar de óxido de estanho dopado com flúor e platina (Pt/FTO) por um eletrodo de $\mathrm{MoS}_{2}$ /papel grafite (GP), aumentando sua eficiência de $6,22 \%$ a 6,48\%, diminuindo o custo de produção e facilitando produção em larga escala. 
Seguindo os estudos de Martins et al. (2018), tem-se ainda a aplicação do grafeno no processo de fotodegradação de poluentes orgânicos, utilizado atualmente com o dióxido de titânio, ao qual por si só possui eficiência reduzida devido a diversos fatores, sendo um deles o seu gap de banda (3.2 ev para anastase e $3.0 \mathrm{ev}$ para fase rutilo) resultar em uma ativação espectral reduzida, correspondendo a apenas $5 \%$ do espectro solar. 0 grafeno, mostrou-se promissor ao ser utilizado na dopagem frente ao $\mathrm{TiO}_{2}$ para a produção de nanocompósitos, aumentando suas propriedades ópticas e fotocatalíticas, ocasionando em melhor adsorção de poluentes como azul de metileno e ciprofloxacino na superfície das nanopartículas.

0 azul de metileno citado anteriormente apresenta enorme problema ao meio ambiente, e algumas das soluções para resolver o problema ocasionado por ele é pelo tratamento através da adsorção. Nesse quesito, os materiais que se mostraram promissores de acordo com a literatura foram os hidrocarvões, onde considerando-se os parâmetros das isotermas de adsorção de Langmuir, o hidrocarvão de resíduo têxtil apresentou capacidade adsortiva de aproximadamente $140,35 \mathrm{mg}^{-1} \mathrm{~g}^{-1}$. Outros materiais da mesma categoria como o hidrocarvão ativado com $\mathrm{NaOH}$ de casca de coco e hidrocarvão de resíduos de acerola apresentaram também considerável capacidade de adsorção, sendo 108,8 mg.g ${ }^{-1}$ e 107,0 mg.g ${ }^{-1}$, respectivamente (Lima et al., 2020). É válido salientar também que a casca de coco é um material lignocelulósico, isto é, um biomaterial que possui em sua composição a celulose, as hemiceluloses e as ligninas, e que por conta desse fator apresenta capacidade adsortiva (Ferreira, 2015).

Porém, segundo o explicitado por Araújo (2018), o OG possui resultados ainda mais satisfatórios usando-se os parâmetros de Langmuir, assumindo mais que o dobro do que capacidade adsortiva frente ao hidrocarvão de resíduo têxtil, sendo 365,14 mg.g-1 para amostras de OG0123 (amostra com $23 \mathrm{~mL}$ de $\mathrm{H}_{2} \mathrm{SO}_{4}$ para $2 \mathrm{~mL}$ de $\mathrm{H}_{2} \mathrm{O}, 3 \mathrm{~h}$ de reação,

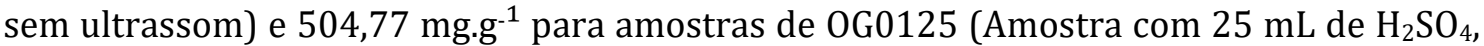
2-3 h de reação, sem ultrassom), apresentando melhor pH de adsorção entre 5 e 6 e remoção de até $99 \%$ da concentração inicial de azul de metileno em 100 mg.L. ${ }^{1}$. 0 mesmo estudo ainda expressa a ação do OG em efluentes têxteis brutos, analisando a remoção da cor e turbidez na relação de $30 \mathrm{~mL}$ de efluente para $1 \mathrm{~mL}$ de suspensão aquosa de OG (na concentração de 0,6 g.L - $^{-1}$ de OG para OG0123 e 0,46 g.L-1 de OG para OG0125) em $1 \mathrm{~h}$ de adsorção, a demanda química de oxigênio (DQO) e a turbidez ficaram em torno de $60 \%$ a $90 \%$, respectivamente, e o percentual de remoção da cor foi de $75 \%$. Conclui-se então que o OG é promissor para a remoção de cor em efluentes, tornando-se economicamente e ambientalmente vantajosas, caso utilizado o Método de Hummers.

Sua utilização se expande para materiais 3D à base de grafeno, valendo-se da adsorção para remover metais pesados do meio ambiente, como descrito por Lai et al. (2019), esse tipo de material causa impacto severo até mesmo em baixas concentrações, tendendo a bioacumular em organismos e persistir dentro da cadeia alimentar. A lista de metais onde há adsorção inclui $\mathrm{Pb}(\mathrm{II}), \mathrm{Cu}(\mathrm{II}), \mathrm{Cr}(\mathrm{VI}), \mathrm{Ni}(\mathrm{II}), \mathrm{Co}(\mathrm{II})$ e $\mathrm{Cd}(\mathrm{II})$, onde microesferas de aerogel a base de OG/Quitosana apresentaram capacidade adsortiva de $747,5 \mathrm{mg} . \mathrm{g}^{-1}$, para $\mathrm{Pb}(\mathrm{II})$, e $457.5 \mathrm{mg} \cdot \mathrm{g}^{-1}$, para $\mathrm{Cu}(\mathrm{II})$, expandindo suas aplicabilidade conforme suas propriedades de textura, área de superfície e estrutura uniforme de poros são otimizadas.

É possível ainda aplicar a adsorção química em outros campos como a limpeza de vazamentos químicos. Um estudo de Bao et al. (2016) concluiu que há um melhor gerenciamento desse tipo de crise quando utiliza-se adsorção para limpeza, evitando também a poluição secundária ocasionada por diluição, lavagem e enterro. Os autores utilizaram miçangas de OG envoltas de uma camada de montmorilonita, relatando que o material é também um retardador de fogo eficiente, um fator que faz diferença na administração desses acidentes. 


\section{Poluição gerada pelo grafeno após seu uso}

É visto que a utilização do grafeno, bem como seus derivados, é possível no contexto do meio ambiente; entretanto, não se pode ignorar os efeitos após o seu descarte. Então, após a análise dos resíduos gerados na sua síntese, bem como as suas possíveis aplicações, outra vertente na qual o grafeno interage com a natureza é no tocante à sua liberação após a fabricação e uso.

A importância de tecnologias sustentáveis é inegável frente à preocupação mundial com o meio ambiente, em face ao avanço expressivo da tecnologia. Quando se trata de nanomateriais, como o grafeno, o termo nanotoxicologia tem tomado relevância, com preocupações quanto à sua biodegradabilidade (Zarbin e Oliveira, 2013). De acordo com Franchi et al. (2012), o estudo e o conhecimento da citotoxicidade e da genotoxicidade de um nanomaterial é indispensável para dimensionar os possíveis impactos ambientais causados pelo seu descarte inadequado, assim como projetar ferramentas para proteção da natureza e das pessoas.

Hu e Zhou (2013) estudaram diversos aspectos sobre a relação entre o grafeno, a saúde humana e o meio ambiente. De acordo com os autores, o conhecimento das interações biológicas do grafeno com os organismos (proteínas, células, metais entre outros) é importante para o desenvolvimento de tecnologias seguras, tornando a biocompatibilidade e nanotoxicidade do grafeno temáticas de suma importância nesse quesito. Nesse âmbito, não foram encontrados muitos trabalhos que propõem-se em estudar a biodegradabilidade do grafeno após seu uso; entretanto, quando se pesquisa sobre essa mesma abordagem para nanomateriais de carbono, grupo no qual o grafeno pertence, encontram-se trabalhos com perspectivas pertinentes.

Katwa et al. (2012) apontam a importância em se discutir acerca do impacto gerado pela exposição humana a nanomateriais de carbono. Os autores estudaram como mastócitos pulmonares e cardiovasculares, células que agem através da variação de sinalizadores biológicos, iriam responder à exposição (via respiratória e injeção intravenosa) a múltiplas camadas de nanotubos de carbono. Eles concluíram que os mastócitos são ativados para regular possíveis toxicidades geradas pelo nanomaterial estudado, tornando possível o direcionamento medicinal dessas células para tratamentos em casos de alta exposição de nanomateriais semelhantes. Diante disso, pode-se notar que o contato humano não controlado com nanomateriais de carbono, como o grafeno, pode impactar diretamente na saúde do indivíduo.

A constatação de Katwa et al. (2012) é assegurada pelo trabalho de Zhao et al. (2017). Em contrapartida aos notórios benefícios tecnológicos exibidos pelo grafeno, segundo Zhao et al. (2017), nanomateriais da família do grafeno merecem um estudo mais aprofundado, por geraram efeitos negativos, tais como: acumulação de produtos peroxidados, formação de radicais livres, resposta inflamatória, entre outros. No entanto, alguns autores defendem que essa nanotoxicidade exibida pelos nanomateriais de carbono está mais relacionado com seu uso do que com sua natureza química.

De acordo com Zarbin e Oliveira (2013), nanomateriais como o grafeno não são inerentemente tóxicos, posto suas estabilidades química (baixa reatividade frente a compostos químicos) e bioquímica (baixa reatividade frente a substâncias biológicas) . No entanto, os autores apontam que modificações nessas nanoestruturas, como ativações químicas ou impurezas, podem propiciar reações bioquímicas indesejáveis. Nesse sentido, Seabra et al. (2013) explanaram o uso de ácido sulfúrico e peróxido de hidrogênio $\left(\mathrm{H}_{2} \mathrm{SO}_{4} / \mathrm{H}_{2} \mathrm{O}_{2}\right)$ para purificação de nanotubos de carbono, viabilizando a remoção de catalisadores residuais, objetivando a degradação do nanomaterial. Assim, os autores relataram que em 8 semanas a nanoestrutura de carbono se reduziu a menos da metade do tamanho original, com degradação total após 16 semanas com esse tratamento. Diante disso, pode-se entender que o mecanismo para decomposição acelerada de nanotubos de carbono centra-se em degradar os compostos residuais aderidos neles. 
No entanto, Ferreira et al. (2019) realizaram um estudo cuja conclusão foi oposta à descrita por Zarbin e Oliveira (2013) e estudada por Seabra et al. (2013). Ferreira et al. (2019) estudaram a toxicidade de OG, com e sem a ativação funcional de grupos amina e óxido de ferro, $\mathrm{OG}_{\mathrm{NH} 2 / \mathrm{Fe}}$ e $\mathrm{OG}$, respectivamente, frente a sementes de Lactuca sativa L. Os autores alegam estudar a toxicidade do $\mathrm{OG}_{\mathrm{NH} 2 / \mathrm{Fe}}$ e $\mathrm{OG}$ sob a possibilidade de utilizar o $\mathrm{OG}$ para tratamento de efluentes têxteis como adsorvente, o que pode gerar o $\mathrm{OG}_{\mathrm{NH} 2 / \mathrm{Fe}}$, isto é, OG contaminado. A escolha da ativação funcional por amina pode ser explicado pelo alto número de corantes com estruturas carbônicas nitrogenadas, tal como o azo (Vecchio, 2019), e o óxido de Fe pelo uso do seu íon em processos de tratamentos de efluentes, como o Fenton (Azevedo et al., 2020).

Sendo assim, segundo Ferreira et al. (2019), o $\mathrm{OG}_{\mathrm{NH} 2 / \mathrm{Fe}}$ exibiu menor toxicidade frente às sementes de Lactuca sativa L. do que o OG, seu precursor. Os autores ressaltaram que são necessárias investigações mais detalhadas sobre essas toxicidades; entretanto, comparando os resultados de Ferreira et al. (2019) e Zarbin e Oliveira (2013) nota-se que a toxicidade do nanomateriais de carbono, como o grafeno, quimicamente ativados pode ser mais relativa às características químicas dos ativadores do que o ativado, 0 nanomaterial.

Visando a investigar a nanotoxicologia do OG em meios aquáticos, Ferreira et al. (2015) compararam os efeitos de três materiais frente ao bioindicador Hydra attenuata, posto que esses organismos são sensíveis a poluentes. Os autores estudaram o grafeno comercial, resíduo de queima de bagaço de cana e nanoestrutura de carvão ativado. Os resultados obtidos mostram que, com $100 \mu \mathrm{g} \cdot \mathrm{mL}^{-1}$ de grafeno, as hidras morreram e/ou desintegraram em $72 \mathrm{~h}$. Já a exposição da hidra aos outros dois materiais apenas resultou em efeitos leves na mesma. Assim, nota-se que o grafeno tem o potencial de afetar ambientes aquíferos, quando mal descartado.

0 estudo feito por Ferreira et al. (2015) pode ser replicado a outras espécies aquáticas, como realizado por Zhang et al. (2017). Esses autores estudaram o impacto da exposição de embriões do peixe-zebra Danio rerio ao OG, em concentrações de 1 a $100 \mu \mathrm{g} . \mathrm{mL}^{-1}$. Foi observado que a exposição dos embriões a esse nanomaterial provocaram modificações genéticas, gerando larvas com más formações, tais como disfunção cardíaca e anomalia em sua estrutura esquelética. Diante desses resultados, os autores sugerem que as administrações governamentais e os produtores deste nanomaterial atuem em defesa do meio ambiente frente ao potencial risco da difusão do OG.

Quando se trata dos resíduos de grafeno despejados no solo, Song et al. (2021) estudaram como a concentração de OG no solo do tipo Cambissolo Háplicos afetaram o crescimento da Larix olgensis, bem como os atributos do solo. As concentrações de OG estudadas foram de 0 a $500 \mathrm{mg} . \mathrm{L}^{-1}$ e as amostras foram incubadas por 30, 40 e 50 dias. Os autores relataram que à baixas concentrações de OG (entre 50 a $100 \mathrm{mg} \cdot \mathrm{L}^{-1}$ ) substâncias importantes para o cultivo dessas plantas sofreram modificações, gerando impactos negativos, como a perda de biomassa dessas mudas. Entretanto, em altas concentrações (250 a 500 mg. $\mathrm{L}^{-1}$ ), o OG colaborou para a redução da matéria orgânica no solo, assim como a inibição da etapa de ciclase do nitrogênio e do fósforo no solo, permitindo sua maior abundância no mesmo, o que contribuiu positivamente com o crescimento das mudas. Assim, observa-se que a relevância em se conhecer métodos eficazes de descarte do grafeno, posto que esse ato pode gerar consequências positivas ou negativas.

Ainda na vertente do plantio $\mathrm{Hu}$ et al. (2014) analisaram o impacto causado pelo concentração de arsênio (As) interagindo com o óxido de grafeno em plantações de trigo. De acordo com os autores, um dos problemas provocados pelo descarte incorreto de nanomateriais no meio ambiente é a sua potencialidade em intensificar características tóxicas de determinadas substâncias, chamada pelos autores de nanotoxicidade indireta do grafeno. Para o trigo, o As é um contaminante, posto seu perfil fitotóxico. De acordo com os autores, quando isolado, o OG não exibia dano ao trigo; entretanto, as amostras de 
OG e As combinadas em proporções de 0,1, 1 e $10 \mathrm{mg} . \mathrm{L}^{-1}$ de óxido para cada $10 \mathrm{mg} . \mathrm{L}^{-1} \mathrm{de}$ As, causaram efeitos graves no trigo, com destaque para o aumento expressivo do superóxido dismutase, que relaciona-se com o sistema de defesa da planta.

Em concordância com os resultados obtidos por Hu et al. (2014), Souza et al. (2018) expuseram o organismo Ceriodaphnia dubia ao óxido de grafeno, em diferentes concentrações. Conforme os autores, essa exposição gerou efeitos crônicos na espécie estudada, como diminuição das taxas de alimentação e reprodução, e o aumento da letalidade. Partindo desses resultados, segundo Souza et al. (2018), demonstra-se a importância da avaliação da nanotoxicologia desses materiais por parte de programas de monitoramento ambiental.

Após analisadas as possíveis consequências nas quais o descarte deste nanomaterial pode gerar, tanto no meio ambiente como na saúde humana, torna-se relevante investigar as razões que formam esse resíduo posterior ao seu uso em tecnologias diversas.

Os resíduos de nanomateriais de carbono podem ser gerados pelo desgaste devido ao uso de materiais nos quais ele compõe, como materiais poliméricos. Baseados nisso, Rhiem et al. (2016) quantificaram a liberação de resíduos de nanotubos de carbonos gerados por diversas condições de descarte, com o intuito de analisar a liberação deste nanomaterial caso o material que o possui na composição seja descartado inadequadamente. As amostras estudadas foram compósitos de policarbonato, que foram impregnadas com nanotubos de carbono-14 $\left({ }^{14} \mathrm{C}\right)$, objetivando localizar este nanomaterial pela radioatividade do isótopo do carbono. Assim, essas amostras foram expostas às seguintes condições: irradiação, exposição a água, a altas e baixas temperaturas, imersão em solução alcalina, chuva ácida simulada, imersão em efluente (mistura de solventes) e, por fim, sua superfície foi atritada. Com isso, os autores relataram uma liberação média de 14,7 $\mu \mathrm{g}$ de nanotubos de carbono, o que evidencia a possibilidade da interação humana indevida ou a exposição ambiental com esse nanomaterial livre.

Schlagenhauf et al. (2014) apresentaram um trabalho de revisão sob a mesma perspectiva de Rhien et al. (2016). Nesse aspecto, Schlagenhauf et al. (2014) estudaram uma gama de polímeros de carbono em três diferentes condições para liberação dos nanotubos de carbono: i) ações mecânicas: arranhar, lixar, furar e serrar; ii) fogo: deposição indevida desse polímeros, com queima acidental, pode levar à decomposição dos nanotubos de carbono, e iii) intemperismo.

Dos 10 trabalhos que analisaram ações mecânicas, Schlagenhauf et al. (2014) relataram que 6 deles não encontraram resíduos de nanotubos de carbono. Já quando se analisou o intemperismo, 5 dos 10 trabalhos analisados mostraram liberação deste nanomaterial, mesmo relatado nos 6 dos 10 trabalhos sobre deposição via queima investigados pelos autores. Diante disso, mesmo após seu uso, nanomateriais de carbono, como o grafeno, merecem atenção quando se trata do seu descarte.

\section{Conclusão}

Diante dos resultados discutidos, vê-se então que os estudos envolvendo grafeno e suas aplicações vêm crescendo ao longo dos anos devido às suas excelentes propriedades físicas e químicas, e que sua interação com o meio ambiente, bem como o impacto da sua síntese neste meio, são pontos importantes para analisar sua viabilidade de uso tanto em âmbito industrial como em aplicações tecnológicas.

No âmbito da síntese, foi visto que o método de crescimento epitaxial gera resíduos de acetona e álcool isopropílico, onde os mesmos podem ser destilados e reutilizados. Já para o método de deposição química, os principais resíduos encontrados são a solução de $\mathrm{HCl}$, a acetona e o PMMA, que podem ser direcionadas para tratamentos de efluentes, destilação ou reciclagem. Não foi observada geração de resíduo para o 
método de separação mecânica. Além disso, foi percebido que na separação química, reagentes em excesso e gases como $\mathrm{NO}_{2}$ e $\mathrm{N}_{2} \mathrm{O}_{4}$ configuraram-se como resíduos, sendo observadas a regulação do $\mathrm{pH}$ e captação dos gases por microalgas como rotas de tratamento adequado.

Já em suas aplicações no que se refere ao meio ambiente, foi visto que o grafeno pode ser usado em células de energia fotovoltaica, com aumento da eficiência dessas células, quando comparados resultados sem o grafeno, além de existir a possibilidade da produção em larga escala. Ademais, constatou-se que o grafeno também pode ser utilizado no processo de tratamentos de efluentes, na fotodegradação de corantes como o azul de metileno.

Por fim, quando se estudou o descarte do grafeno após seu uso, o presente trabalho permitiu concluir que muitos estudos focam na nanotoxicidade e biodegradabilidade desse nanomaterial. Foi percebido que o ser humano, bem como seres aquáticos e plantas, são sensíveis ao grafeno, tal como visto para mastócitos pulmonar humano, a espécie Hydra attenuata e as sementes de Lactuca sativa L. Além disso, na avaliação de trabalhos que analisaram processos nos quais o grafeno é depositado no meio ambiente, foi constatado que essa deposição é devido a ações da natureza sobre o material, como arranhões, lixamentos, furos etc.

\section{Conflito de interesses}

Os autores declaram não haver conflito de interesses.

\section{Referências}

Araújo, C. M. B. Estudo da produção e aplicação de óxidos de grafite e grafeno para remoção de contaminantes em meio aquoso. Recife: Universidade Federal de Pernambuco, 2018. (Dissertação de mestrado).

Azevedo, P. G. F.; Oliveira, D. C. S.; Cavalcanti, L. A. P. Processos físicos e químicos para o tratamento de efluentes: uma revisão integrativa. Revista Brasileira de Gestão $\begin{array}{lllll}\text { Ambiental e Sustentabilidade, v. 7, } & \text { n. 17, } & \text { p. 1667-1678, }\end{array}$ https://doi.org/10.21438/rbgas(2020)071740

Bao, C.; Bi, S.; Zhang, H.; Zhao, J. Graphene oxide beads for fast clean-up of hazardous chemicals. Journal of Materials Chemistry A, v. 4, n. 24, p.9321-9702, 2016. https://doi.org/10.1039/C6TA01411A

Camargos, J. S. F.; Semmer, A. O.; Silva, S. N. Características e aplicações do grafeno e do óxido de grafeno e as principais rotas para síntese. The Journal of Engineering and Exact Sciences, v. 3, n. 8, 2017. https://doi.org/10.18540/jcecvl3iss8pp1118-1130

Castro, M. O. Síntese de grafeno pelo método CVD. Fortaleza: Universidade Federal do Ceará, 2011. (Dissertação de mestrado).

Chen, D.; Zhang, H.; Liu, Y.; Li, J. Graphene and its derivatives for the development of solar cells, photoelectrochemical, and photocatalytic applications. Energy \& Environmental Science, v. 6, n. 5, p. 1341-1642, 2013. https://doi.org/10.1039/C3EE23586F

Coletti, G. F.; Tavaresa, G. A.; Bendassolli, J. A. Recuperação de acetona em resíduos laboratoriais: uma abordagem sobre aspectos da gestão, operacionais e da eficiência ambiental. Química Nova, v. 42, n. 6, 2019. https://doi.org/10.21577/01004042.20170361 
Cordeiro, G. L.; Oliveira, R. R.; Ferreira, N. A. M.; Xavier, G. L.; Yoshito, W. K.; Lima, N. B.; Lazar, D. R. R.; Ussui, V. Síntese química e caracterização de grafeno. Anais do 59o Congresso Brasileiro de Cerâmica, São Paulo, 2015.

Costa, M. C. F. Grafeno e seus derivados: estudo das propriedades de superfície e processos de transferência para substratos poliméricos. São Paulo: Universidade Presbiteriana Mackenzie, 2017. (Dissertação de mestrado).

Ferreira S. B.; Vallim, J. H.; Clemente, Z.; Martinez, D.; Castro, V. L. Avaliação ecotoxicológica de nanomateriais de carbono em Hydra attenuata. Anais do 9o Congresso Interinstitucional de Iniciação Científica, Campinas, 2015.

Ferreira, B. C. S. Desenvolvimento de novos materiais lignocelulósicos e quitinosos com potencial aplicação em química ambiental. Belo Horizonte: Universidade Federal de Minas Gerais, 2015. (Tese de doutorado).

Ferreira, L. E. L.; Souza, Z. S. B.; Fraga, T. J. M.; Carvalho, M. N.; Motta Sobrinho, M. A. Avaliação da toxicidade do nanoadsorvente óxido de grafeno amino- $\mathrm{Fe}_{3} \mathrm{O}_{4}$-funcionalizado em sementes de Lactuca sativa L. Anais do $30^{\circ}$ Congresso Brasileiro de Engenharia Sanitária e Ambiental, Natal, 2019.

Franchi, L. P.; Santos, R. A.; Matsubara, E. Y.; Lima, J. C.; Rosolen, J. M.; Takahashi, C. S. Citotoxicidade e genotoxicidade de nanotubos de carbono. Química Nova, v. 35, n. 3, p. 571-580, 2012. https://doi.org/10.1590/S0100-40422012000300025

Hu, X.; Kang, J.; Lu, K.; Zhou, R.; Mu, L.; Zhou, Q. Graphene oxide amplifies the phytotoxicity of arsenic in wheat. Scientific Reports, v. 4, n. 6122, 2014. https://doi.org/10.1038/ srep06122

$\mathrm{Hu}, \mathrm{X}$; Zhou, Q. Health and ecosystem risks of graphene. Chemical Reviews, v. 113, n. 5, p. 3815-3835, 2013. https://doi.org/10.1021/cr300045n

Huang, N.; Li, G.; Xia, Z.; Zheng, F.; Huang, H.; Li, W.; Xiang, C.; Su, Y.; Sun, P.; Sun, X. Solution-processed relatively pure $\mathrm{MoS}_{2}$ nanoparticles in-situ grown on graphite paper as an efficient FTO-free counter electrode for dye-sensitized solar cells. Electrochimica Acta, v. 235, p. 182-190, 2017. https://doi.org/10.1016/j.electacta.2017.02.111

Katwa, P.; Wang, X.; Urankar, R. N.; Podila, R.; Hilderbrand, S. C.; Fick, R. B.; Rao, A. M.; Ke, P. C.; Wingard, C. J.; Brown, J. M. A Carbon nanotube toxicity paradigm driven by mast cells and the IL-33/ST 2 axis. Small, v. 8, n. 18, p. 2904-2912, 2012. https://doi.org/10.1002/ smll.201200873

Lai, K. C.; Lee, L. Y.; Hiew, B. Y. Z.; Thangalazhy-Gopakumar, S.; Gan, S. Environmental application of three-dimensional graphene materials as adsorbents for dyes and heavy metals: Review on ice-templating method and adsorption mechanisms. Journal of Environmental Sciences, v.79, p.174-199, 2019. https://doi.org/10.1016/ j.jes.2018.11.023

Leal, N. N. S. Mapeamento da condutividade de camadas de grafeno de diferentes espessuras usando a microscopia de força atômica condutora e espectroscopia Raman. Maceió: Universidade Federal de Alagoas, 2016. (Dissertação de mestrado).

Lima, A. F.; Fagnani, H. M. C.; Santos, W. L. F.; Barros M. A. S. D. Adsorção de azul de metileno em hidrocarvões de resíduos têxteis. Matéria, v. 25, n. 4, 2020. https://doi.org/10.1590/S1517-707620200004.1185

Marion, B.; Hasan, N. Grafeno: inovações, aplicações e sua comercialização. Interfaces Científicas - Exatas e Tecnológicas, v. 2, n. 1, p. 29-40, 2016. https://doi.org/10.17564/ 2359-4934.2016v2n1p29-40 
Martinez, J.G. B. Modelagem e simulação da fixação de emissões de grupo motogerador por cultivo de microalgas em fotobiorreatores industriais. Curitiba: Universidade Federal do Paraná, 2018. (Dissertação de mestrado).

Martins, P. M.; Ferreira, C. G.; Silva, A. R.; Magalhães, B.; Alves, M. M.; Pereira, L.; Marques, P. A. A. P.; Melle-Franco, M.; Lanceros-Méndez, $\mathrm{S}$. $\mathrm{TiO}_{2}$ /graphene and $\mathrm{TiO}_{2}$ /graphene oxide nanocomposites for photocatalytic applications: A computer modeling and experimental study. Composites Part B: Engineering, v. 145, p. 39-46, 2018. https://doi.org/10.1016/ j.compositesb.2018.03.015

Nunes, M. M. Otimização dos métodos de síntese do grafeno como catalisador para aplicações na área aeroespacial. São Paulo: Instituto Nacional de Pesquisas Espaciais, 2019. (Relatório final de projeto).

Oliveira, A. R. Obtenção de filmes de multicamadas de grafeno utilizando Dip-Coating. Uberaba: Universidade Federal do Triângulo Mineiro, 2014. (Dissertação de mestrado).

Rhiem, S.; Barthel, A. K.; Plath, A. M.; Henning, M. P.; Wachtendorf, V.; Sturm, H.; Schäffer, A.; Maes, H. M. Release of ${ }^{14} \mathrm{C}$-labelled carbon nanotubes from polycarbonate composites. Environmental Pollution, v. 215, p.356-365, 2016. https://doi.org/10.1016/ j.envpol.2016.04.098

Rocha, J. P. M. Grafeno como fonte de energia renovável: um estudo prospectivo. Brasília: Universidade de Brasília, 2015. (Trabalho de conclusão de curso).

Rodrigues, A.; Neves, F. A.; Silva, O.; Pedro, R. Resíduos químicos: gestão e possíveis tratamentos. Araraquara: Universidade Estadual Paulista, 2010. (Trabalho de conclusão de curso).

Roma, J. C. Os Objetivos de Desenvolvimento do Milênio e sua transição para os Objetivos de Desenvolvimento Sustentável. Ciência e Cultura, v. 71, n. 1, p. 33-39, 2019. https://doi.org/10.21800/2317-66602019000100011

Sá, T. G. M. Crescimento de "multicamadas" de grafeno epitaxial em substratos de SiC à pressão atmosférica. Belo Horizonte: Universidade Federal de Minas Gerais, 2011. (Dissertação de mestrado).

Schlagenhauf, L.; Nuesch, F.; Wang, J. Release of carbon nanotubes from polymer nanocomposites. Fibers, v. 2, p. 108-127, 2014. https://doi.org/10.3390/fib2020108

Seabra, A. B.; Paula, A. J.; Durán, N. Redox-enzymes, cells and micro-organisms acting on carbon nanostructures transformation: A mini-review. Biotechnology Program, v. 29, p. 1, 2013. https://doi.org/10.1002/btpr.1673

Silva, P. A. P. Nanocompósito de polipropileno reforçado com grafeno. Belo Horizonte: Centro Federal de Educação Tecnológica de Minas Gerais, 2016. (Trabalho de conclusão de curso).

Song, J.; Luo, N.; Sang, Y.; Duan, C.; Cui, X. Graphene oxide affects growth and physiological indexes in Larix olgensis seedlings and the soil properties of Haplic Cambisols in Northeast China. Environmental Science and Pollution Research, v. 28, p. 20869-20882, 2021. https://doi.org/10.1007/s11356-020-11972-w

Souza, J. P.; Venturini, F. P.; Santos, F.; Zucolotto, V. Chronic toxicity in Ceriodaphnia dubia induced by graphene oxide. Chemosphere, v.190, p.218-224, 2018. https://doi.org/10.1016/j.chemosphere.2017.10.018 
Vecchio, P. D. Degradação de contaminantes de diferentes classes por processos oxidativos avançados: $\mathrm{O}_{3}, \mathrm{O}_{3} / \mathrm{UV}$ e $\mathrm{O}_{3} / \mathrm{Al}_{2} \mathrm{O}_{3}$. Porto Alegre: Universidade Federal do Rio Grande do Sul, 2019. (Dissertação de mestrado).

Vidales, L. T.; Kindlein Júnior, W.; Cândido, L. H. A.; Demori, R.; Mauler, R. S. Blendas PC/PMMA como alternativa para reciclagem de óculos apreendidos pela Receita Federal do Brasil. Anais do XII Congresso Brasileiro de Polímeros, Associação Brasileira de Polímeros, Florianópolis, 2013.

Vieira Segundo, J. E. D.; Vilar, E. O. Grafeno: Uma revisão sobre propriedades, mecanismos de produção e potenciais aplicações em sistemas energéticos. Revista Eletrônica de Materiais e Processos, v. 11, n. 2, p. 54-57, 2016.

Zarbin, A. J. G.; Oliveira, M. M. Nanoestruturas de carbono (nanotubos, grafeno): Quo vadis? Química Nova, v. 36, n. 10, p. 1533-1539, 2013. https://doi.org/10.1590/S010040422013001000009

Zhang, X.; Zhou, Q.; Zou, W.; Hu, X. Molecular mechanisms of developmental toxicity induced by graphene oxide at predicted environmental concentrations. Environmental Science \& Technology, v. 51, n. 14, p. 7861-7871, 2017. https://doi.org/10.1021/ acs.est.7b01922

Zhao, J.; Cao, X.; Wang, Z.; Dai, Y.; Xing, B. Mechanistic understanding toward the toxicity of graphene-family materials to freshwater algae. Water Research, v. 111, p. 18-27, 2017. https://doi.org/10.1016/j.watres.2016.12.037

Informação da Licença: Este é um artigo Open Access distribuído sob os termos da Licença Creative Commons
(CC) Attribution, que permite uso irrestrito, distribuição e reprodução em qualquer meio, desde que a obra original seja devidamente citada. 